\title{
Towards a fasting-mimicking diet for critically ill patients: the pilot randomized crossover ICU-FM-1 study
}

Lisa Van Dyck', Ilse Vanhorebeek ${ }^{1}$, Alexander Wilmer², An Schrijvers', Inge Derese ${ }^{1}$, Liese Mebis ${ }^{1}$, Pieter J. Wouters ${ }^{1}$, Greet Van den Berghe ${ }^{1}$, Jan Gunst ${ }^{1+}$ and Michaël P. Casaer $^{1{ }^{*+}} \mathbb{0}$

\begin{abstract}
Background: In two recent randomized controlled trials, withholding parenteral nutrition early in critical illness improved outcome as compared to early up-to-calculated-target nutrition, which may be explained by beneficial effects of fasting. Outside critical care, fasting-mimicking diets were found to maintain fasting-induced benefits while avoiding prolonged starvation. It is unclear whether critically ill patients can develop a fasting response after a short-term nutrient interruption. In this randomized crossover pilot study, we investigated whether 12-h nutrient interruption initiates a metabolic fasting response in prolonged critically ill patients. As a secondary objective, we studied the feasibility of monitoring autophagy in blood samples.

Methods: In a single-center study in 70 prolonged critically ill patients, 12-h up-to-calculated-target feeding was alternated with 12-h fasting on day $8 \pm 1 \mathrm{in} \mathrm{ICU}$, in random order. Blood samples were obtained at the start of the study, at the crossover point, and at the end of the 24-h study period. Primary endpoints were a fasting-induced increase in serum bilirubin and decrease in insulin requirements to maintain normoglycemia. Secondary outcomes included serum insulin-like growth factor I (IGF-I), serum urea, plasma beta-hydroxybutyrate (BOH), and mRNA and protein markers of autophagy in whole blood and isolated white blood cells. To obtain a healthy reference, mRNA and protein markers of autophagy were assessed in whole blood and isolated white blood cells of 23 matched healthy subjects in fed and fasted conditions. Data were analyzed using repeated-measures ANOVA, Fisher's exact test, or Mann-Whitney $U$ test, as appropriate.
\end{abstract}

Results: A 12-h nutrient interruption significantly increased serum bilirubin and $\mathrm{BOH}$ and decreased insulin requirements and serum IGF-I (all $p \leq 0.001$ ). Urea was not affected. BOH was already increased from $4 \mathrm{~h}$ fasting onwards. Autophagic markers in blood samples were largely unaffected by fasting in patients and healthy subjects.

Conclusions: A 12-h nutrient interruption initiated a metabolic fasting response in prolonged critically ill patients, which opens perspectives for the development of a fasting-mimicking diet. Blood samples may not be a good readout of autophagy at the tissue level.

(Continued on next page)

\footnotetext{
* Correspondence: michael.casaer@uzleuven.be

${ }^{\dagger} J$ an Gunst and Michaël P. Casaer contributed equally to this work.

'Department of Cellular and Molecular Medicine, Clinical Division and

Laboratory of Intensive Care Medicine, KU Leuven, Herestraat 49, B-3000

Leuven, Belgium

Full list of author information is available at the end of the article
}

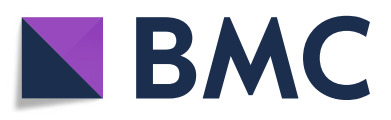

(c) The Author(s). 2020 Open Access This article is licensed under a Creative Commons Attribution 4.0 International License, which permits use, sharing, adaptation, distribution and reproduction in any medium or format, as long as you give appropriate credit to the original author(s) and the source, provide a link to the Creative Commons licence, and indicate if changes were made. The images or other third party material in this article are included in the article's Creative Commons licence, unless indicated otherwise in a credit line to the material. If material is not included in the article's Creative Commons licence and your intended use is not permitted by statutory regulation or exceeds the permitted use, you will need to obtain permission directly from the copyright holder. To view a copy of this licence, visit http://creativecommons.org/licenses/by/4.0/. The Creative Commons Public Domain Dedication waiver (http://creativecommons.org/publicdomain/zero/1.0/) applies to the data made available in this article, unless otherwise stated in a credit line to the data. 
(Continued from previous page)

Trial registration: ISRCTN, ISRCTN98404761. Registered 3 May 2017.

Keywords: Intensive care, Critical illness, Nutrition, Intermittent fasting, Metabolism, Autophagy

\section{Background}

For a long time, early artificial nutrition has been assumed to benefit critically ill patients, since prevention of a caloric deficit repeatedly has been associated with improved outcome [1-3]. However, recent large randomized controlled trials (RCTs) have shown that early enhanced feeding did not benefit critically ill patients, and two RCTs-the EPaNIC and PEPaNIC RCT-found that early supplementation of insufficient or contraindicated enteral nutrition by parenteral nutrition may even increase morbidity as compared to withholding parenteral nutrition until 1 week after intensive care unit (ICU) admission [4-9]. Hypothesis-generating, detailed, secondary analyses of both RCTs associated any feeding dose above the lowest dose with progressively increased harm, which suggests that the negative impact of early parenteral nutrition was dose-related and not related to the route of feeding $[10,11]$. In line with this, two recent RCTs-the CALORIES and Nutrirea-2 RCTfound no outcome difference between the enteral and parenteral route of feeding when macronutrients were provided at isocaloric doses [12, 13]. Altogether, these data suggest that early enhanced nutritional support did not benefit critically ill patients and that relative macronutrient restriction may even be beneficial. Potential protective mechanisms of relative macronutrient restriction include activated autophagy and enhanced ketogenesis, among others, as put forward by experimental models and/or studies on human samples [1417].

Although components of the fasting response may be beneficial in critical illness, prolonged starvation likely comes at a price [18]. A potential alternative to bring about fasting-induced benefits in critically ill patients is the design of a fasting-mimicking diet. Such diets substantially restrict food intake for several hours or days, which is alternated with periods of unlimited intake. Numerous animal studies in various models have demonstrated increased lifespan and protection against agerelated diseases by implementation of such diet [19]. Interestingly, as compared to regular diets, fastingmimicking diets were able to provide the full caloric target, while maintaining an improved metabolic profile during the period of full feeding. Since critical illness shows several parallels with normal aging, including increased cellular stress and accumulation of cellular damage, we hypothesized that a fasting-mimicking diet may be beneficial for critically ill patients as well $[11,20]$.
Until now, fasting-mimicking diets have not been tested in critically ill patients. Moreover, it remains unclear whether critically ill patients can develop a full metabolic fasting response. Indeed, ketogenesis may be impaired in critically ill patients, and the catabolic state induced by severe illness may preclude any additional fasting response [21-23]. Moreover, even if critically ill patients could develop a full fasting response, it remains unclear how long feeding should be restricted before such response would initiate. Although prolonged fasting intervals are theoretically more likely to induce a fasting response, a higher hourly feeding dose and associated fluid load would be needed to avoid underfeeding in such scenario. In this pilot crossover study, we investigated whether 12-h macronutrient restriction is sufficient to initiate a metabolic fasting response in prolonged critically ill patients. In healthy individuals, such response is characterized by increases in ketogenesis, serum bilirubin and urea, decreases in insulin concentrations and insulin-like growth factor I, and activation of autophagy $[4,19,24-26]$. Since it was unclear whether critically ill patients can initiate a full metabolic fasting response, the primary endpoints were based on fasting-induced alterations that were previously shown to be significantly impacted by withholding parenteral nutrition in the first week in ICU [4]. The study was meant as the first "dose-finding" step in the design of a fasting-mimicking diet for critically ill patients, to bring about an optimized metabolic profile and eventually to preserve cellular integrity through autophagy activation. If the pilot study would suggest feasibility, the subsequent aim would be to test such diet in a large RCT powered for clinical endpoints. In preparation of such future studies, we also investigated the feasibility of monitoring autophagy in blood samples as a less invasive surrogate for muscle biopsies.

\section{Methods}

\section{Study design and participants}

This study was a prospective, randomized, crossover pilot study performed in six medical/surgical ICUs at the University Hospitals of Leuven, Belgium. The study was performed in accordance with the principles of the Declaration of Helsinki. The Ethical Committee Research $\mathrm{UZ/KU}$ Leuven approved the protocol and consent forms (S59328). All patients or their legal representatives provided written informed consent before randomization. 
Patients were recruited from August 28, 2017, until July 11, 2018.

All adult patients (18 years or older) who were still in the ICU on day 6 were screened for eligibility. Patients were eligible if they were expected to stay in the ICU and dependent on both mechanical respiratory support and pharmacological and/or mechanical hemodynamic support for at least another $72 \mathrm{~h}$. Patients were excluded if they had serum bilirubin concentrations above $5 \mathrm{mg} /$ $\mathrm{dl}$, were enrolled in another RCT, were considered moribund or had formal therapy restriction as part of advanced care planning, were pregnant or lactating, had any oral intake, were previously enrolled in the study, or were unable to be fasted or fed for 12 consecutive hours (e.g., due to specific need for glucose-containing infusions or due to procedure-related fasting). After having obtained informed consent on day $7 \pm 1$, patients still meeting the inclusion criteria were randomized to receive $50 \%$ of their calculated daily caloric target over 12 consecutive hours, followed by 12 -h fasting (feeding-fasting) on day $8 \pm 1$, or vice versa (fasting-feeding). Day $8 \pm$ 1 was chosen as the intervention day, as our common practice involves withholding parenteral nutrition in the first week in ICU, implying relative macronutrient restriction in the acute phase of illness. Thereby, inclusion of patients in the first week in ICU would have led to a minor difference in caloric intake between the fasting and feeding interval, which would have inflated the chance of false-negative results. Moreover, if the project would ultimately show that fasting-mimicking diets are beneficial, this would be particularly relevant for patients with prolonged ICU stay. Patients were stratified according to admission diagnosis (cardiac surgery versus others) and randomization was performed with use of a central computer in permuted blocks of 10 per stratum. The block size was unknown to the treating physicians and nurses.

During the first week in ICU, all patients received enteral nutrition if possible, which was increased according to tolerance. No parenteral nutrition was administered in the first week after ICU admission as per the centers' nutrition protocol. During ICU stay, all patients received intravenous supplements of trace elements and vitamins, which was continued until the patient received the full calculated caloric target through EN and oral intake (Additional table 1). Blood phosphate, potassium, and magnesium were monitored daily, in order to allow prevention and/or early detection of deficiencies and refeeding syndrome. All patients received blood glucose control targeting $80-110 \mathrm{mg} / \mathrm{dl}$ with insulin throughout ICU stay until they were able to eat by mouth. To that purpose, arterial blood glucose concentrations were monitored every 1 to $4 \mathrm{~h}$ with a blood gas analyzer, and insulin dose was adjusted through continuous intravenous infusion.
On the intervention day at day $8 \pm 1$, during the 12 -h feeding interval, patients received artificial nutrition to cover $100 \%$ of their hourly calculated caloric goal. Nutrition primarily consisted of enteral nutrition, but was supplemented with parenteral nutrition if enteral nutrition was insufficiently tolerated or contraindicated. The enteral and parenteral nutrition formulas prescribed at the discretion of the treating physician are listed in Additional table 2. The nutritional target was calculated and based on corrected ideal body weight, age, and gender (Additional table 1). During the 12-h fasting interval, artificial nutrition was stopped, including any infusion of glucose-containing maintenance infusions. An exception was made for calories from propofol and intravenously administered drugs requiring mandatory dilution in glucose. The insulin infusion rate was preemptively reduced at the start of the 12-h fasting interval to reduce the risk of hypoglycemia. Patients received non-glucose containing fluids in order to provide adequate hydration at the discretion of the treating physician. If a patient developed hypoglycemia, a glucose bolus and/or a glucosecontaining maintenance infusion were given at the discretion of the treating physician.

At the start of the study intervention $(8: 00 \mathrm{~h})$, at the crossover point after $12 \mathrm{~h}(20: 00 \mathrm{~h})$, and at the end of the intervention (after $24 \mathrm{~h}$, at 8:00 $\mathrm{h}$ the following morning), an arterial blood sample was taken. Beta-hydroxybutyrate $(\mathrm{BOH})$ concentrations were measured at the bedside every $4 \mathrm{~h}$ using a point-of-care ketometer (StatStrip Glucose/Ketone Xpress $2^{\circ}$ Meter, Nova Biomedical, provided by Menarini Diagnostics).

To obtain a healthy reference range for autophagic markers in white blood cells and whole blood, healthy volunteers were recruited with similar age, gender, and BMI distributions as the patients (Table 1). Volunteers were instructed to fast for at least $12 \mathrm{~h}$ overnight prior to blood sampling in the morning (fasted state). A second blood sample was obtained $2 \mathrm{~h}$ after a copious breakfast (fed state).

\section{Data collection}

We collected demographical data and daily records of clinical parameters and treatments from the patient data management system (MetaVision Suite, iMDsoft). Upon ICU admission, we quantified severity of illness according to the Acute Physiology and Chronic Health Evaluation II score (APACHE II), and nutritional risk according to the Nutritional Risk Screening score (NRS), and scored sepsis according to the Sepsis-3 criteria [27]. To quantify severity of illness at inclusion in the study, the Sequential Organ Failure Assessment (SOFA) score was calculated over the $24 \mathrm{~h}$ preceding the intervention day. On the intervention day, we collected detailed data regarding nutrition, blood glucose, insulin requirements, 
Table 1 Baseline characteristics of participants

\begin{tabular}{|c|c|c|c|}
\hline Baseline characteristic & $\begin{array}{l}\text { Fasting-feeding } \\
N=35\end{array}$ & $\begin{array}{l}\text { Feeding-fasting } \\
N=35\end{array}$ & $\begin{array}{l}\text { Controls } \\
N=23\end{array}$ \\
\hline Age-median [IQR] & $63.2[51.5-72.8]$ & $67.7[57.6-74.4]$ & $69.2[55.8-79.4]$ \\
\hline Male gender-n (\%) & $18(51.4)$ & $18(51.4)$ & $10(43.5)$ \\
\hline BMl—median [IQR] & $25.2[23.3-31.6]$ & $24.6[21.7-27.1]$ & $27.3[24.3-29.4]$ \\
\hline Admission to surgical ICU—n (\%) & $22(62.9)$ & $22(62.9)$ & NA \\
\hline Cardiac surgery-n (\%) & $7(20.0)$ & $6(17.1)$ & NA \\
\hline Emergency admission—n (\%) & $32(91.4)$ & $31(88.6)$ & NA \\
\hline Sepsis upon ICU admission-n (\%) & $19(54.3)$ & $20(57.1)$ & NA \\
\hline APACHE $\|-$ median [IQR] & $33[27-35]$ & $31[25-36]$ & NA \\
\hline NRS score-median [IQR] & $4[3-5]$ & $4[4-5]$ & NA \\
\hline Diabetes-n (\%) & $7(20.0)$ & $8(22.9)$ & $3(13.0)$ \\
\hline History of malignancy $-n(\%)$ & $10(28.6)$ & $10(28.6)$ & $3(13.0)$ \\
\hline Pre-admission dialysis—n (\%) & $2(5.7)$ & $0(0.0)$ & $0(0.0)$ \\
\hline Study day—median [IQR] & 8 [8-10] & 8 [8-9] & NA \\
\hline SOFA score on randomization day-median [IQR] & $8[6-10]$ & $7[6-11]$ & NA \\
\hline
\end{tabular}

Abbreviations: IQR interquartile range, BMI body mass index, ICU intensive care unit, APACHE Acute Physiology and Chronic Health Evaluation, NRS Nutritional Risk Screening, SOFA Sequential Organ Failure Assessment

and propofol administration in each 12 -h period. Energy from parenteral nutrition included total calories from parenteral nutrition solutions, glucose-containing fluids, and propofol. Severe hypoglycemia was defined as arterial blood glucose concentration below $40 \mathrm{mg} / \mathrm{dl}$.

We recorded the development of new infections and the persistent need for hemodynamic support, respiratory support and renal replacement therapy at day 7 after randomization or at ICU discharge if patients were discharged earlier. Hemodynamic support was defined as mechanical or pharmacological (epinephrine, norepinephrine, dobutamine, dopamine, or vasopressin in any dose) support, and mechanical respiratory support was defined as any ventilation method with positive pressure generation. Patients who died within 7 days after the intervention were marked as persistently dependent on hemodynamic and respiratory support. We recorded mortality within 7 days after randomization, ICU mortality, and mortality within 90 days after randomization. The cause of death was recorded for all patients who died within 90 days after randomization.

\section{Outcome measures}

The primary endpoints were a fasting-induced increase in total serum bilirubin and a decreased insulin need to maintain normoglycemia. The choice of the primary endpoints was based on well-documented fasting-associated alterations in healthy individuals and in critically ill patients in whom parenteral nutrition was withheld until 1 week after ICU admission [4, 18, 25]. Secondary outcomes were changes in plasma and blood ketone concentrations, in serum insulin-like growth factor (IGF-I), and in serum urea. These parameters are similarly affected by short-term fasting in healthy humans, except for urea that shows slower kinetics $[18,19,26]$. Tertiary, exploratory endpoints included the mRNA and protein expression of commonly used autophagic markers in whole blood and isolated white blood cells.

\section{Lab analyses}

Total serum bilirubin was measured with a commercially available assay (Total Bilirubin Reagent, ThermoFisher) and standards with known bilirubin concentrations (Bilirubin Standard Kit, Verichem). Serum urea and plasma betahydroxybutyrate $(\mathrm{BOH})$ were measured with commercially available colorimetric assays (Urea Assay Kit, Cell Biolabs Inc.; and EnzyChrom Ketone Body Assay kit, BioAssay Systems). Serum IGF-I was measured with a commercially available enzyme-linked immunosorbent assay (Human IGF-I Quantikine ELISA kit, R\&D Systems).

Markers of autophagy were investigated in whole blood and in isolated white blood cells. Whole blood was collected in PAXgene ${ }^{\mathrm{Tm}}$ Blood RNA tubes (PreAnalytiX) and was stored at $-80{ }^{\circ} \mathrm{C}$ until RNA extraction with PAXgene ${ }^{\text {m }}$ Blood RNA kits (PreAnalytiX). White blood cells were isolated within $2 \mathrm{~h}$ after blood sampling. Red blood cells were first removed via an osmotically active lysis buffer $\left(\mathrm{NH}_{4} \mathrm{Cl} 8.29 \mathrm{~g} / \mathrm{l}\right.$, EDTA disodium $2-\mathrm{H}_{2} \mathrm{O}$ $\left.0.0372 \mathrm{~g} / \mathrm{l}, \mathrm{KHCO}_{3} 1 \mathrm{~g} / \mathrm{l}\right)$. Subsequently, white blood cells were lysed and stored at $-80^{\circ} \mathrm{C}$ until simultaneous extraction of RNA and proteins with NucleoSpin ${ }^{\circ}$ RNA/ protein filters (Macherey-Nagel). Four hundred fifty nanograms of mRNA of the PAXgene tubes and $210 \mathrm{ng}$ mRNA of the isolated white blood cells were reverse 
transcribed to cDNA. Real-time PCR (StepOne Plus, Applied Biosystems) for gene expression analysis was performed with use of TaqMan chemistry (Applied Biosystems, Hs00223937_m1 for atg3, Hs00169468_ $\mathrm{m} 1$ for atg5, Hs00893766_m1 for atg7, Hs00177654 $\mathrm{m} 1$ for $p 62)$. Relative gene expression was determined with the $2-\Delta \Delta \mathrm{Ct}$ method using beta-2 microglobulin (B2M, Hs00187842_m1) as housekeeping gene. Western blotting was performed on proteins extracted from isolated white blood cells with primary antibodies against LC3 (Sigma, L7543) and p62 (Novus Biologicals, H00008878-M01) and against actin (Abcam, ab3280) as housekeeping protein. Secondary horseradish peroxidaseconjugated antibodies were purchased from DakoCytomation. Blots were visualized with the G:BOX Chemi XRQ (SynGene) and analyzed with SynGene software. Both real-time PCR and western blot data were expressed relative to the median of the 23 fed healthy controls.

\section{Statistical analyses}

We calculated that a sample size of 70 patients, in a crossover design using repeated measures ANOVA, with an alpha level of 0.05 , would be able to detect a $0.3 \mathrm{mg} /$ $\mathrm{dl}$ increase in serum bilirubin concentrations with 93\% power and a decrease of $40 \mathrm{IU}$ per $24 \mathrm{~h}$ in insulin requirements to maintain normoglycemia with $99 \%$ power [28]. The expected effect size and total variance for the primary endpoints $(f=0.169$ for serum bilirubin, $f=$ 0.3055 for insulin requirements) were based on changes observed in the EPaNIC RCT [4], during which nutrition was abruptly increased from day 7 to day 8 in a subset of patients.

Variables were summarized as frequencies and percentages, medians and interquartile ranges, or means and standard errors of the mean, as appropriate. Data were compared with use of repeated measures ANOVA, Fisher's exact test, or Mann-Whitney $U$ test, as appropriate. Where needed, data were transformed with a square root to obtain a near-normal distribution. All statistical analyses were performed with JMP Pro (v14.0.0, SAS Institute). Power was calculated in G*Power (version 3.1.9, Heinrich Heine Universität Düsseldorf). Two-sided $p$ values $\leq 0.05$ were considered to indicate statistical significance, without correction for multiple comparisons.

\section{Results}

\section{Patients and intervention}

On day 6, 1071 patients were screened for eligibility. Reasons for exclusion are shown in Fig. 1. On day $8 \pm 1$, 70 randomized patients received the crossover intervention ( $n=35$ in each group). Baseline characteristics and calculated caloric targets were comparable for both groups (Table 1 and Additional table 3). Over the entire intervention day, patients received similar amounts of total calories $(p=0.65)$, consisting of similar amounts of enteral $(p=0.82)$ and parenteral calories $(p=0.30)$ (Fig. 2 and Additional table 3). During the 12-h feeding interval, patients received a median of $0.95 \mathrm{kcal} / \mathrm{kg} / \mathrm{h}$ (interquartile range (IQR) $0.80-1.15 \mathrm{kcal} / \mathrm{kg} / \mathrm{h}$ ) and during the 12$\mathrm{h}$ fasting interval $0.03 \mathrm{kcal} / \mathrm{kg} / \mathrm{h}$ (IQR $0.00-0.06 \mathrm{kcal} / \mathrm{kg} /$ h). Patients of both randomization groups received comparable amounts of calories during the feeding interval $(p=0.11)$, whereas during the fasting interval, patients in the feeding-fasting group received slightly more calories with a median of $0.04 \mathrm{kcal} / \mathrm{kg} / \mathrm{h}$ (IQR $0.00-0.11 \mathrm{kcal} / \mathrm{kg} /$ $\mathrm{h})$ as compared to the fasting-feeding group with a median of $0.00 \mathrm{kcal} / \mathrm{kg} / \mathrm{h}$ (IQR $0.00-0.01 \mathrm{kcal} / \mathrm{kg} / \mathrm{h}) \quad(p=$ $0.0004)$. In the total study population, administered calories corresponded to $108.1 \%$ of the calculated caloric target (IQR 99.0-119.9\%) during the feeding interval and $0.3 \%$ of the calculated caloric target (IQR $0.0-7.2 \%$ ) during the fasting interval (Fig. 2). Over the entire study day, patients reached $55.8 \%$ of their calculated caloric target (IQR 51.2-62.8\%).

\section{Metabolic response to $12-\mathrm{h}$ fasting versus feeding}

Both serum total bilirubin and insulin requirements were significantly affected by the intervention (interaction $p=$ 0.002 for serum total bilirubin, interaction $p<0.0001$ for insulin requirements, Fig. 3). Regardless of randomization for order, 12-h fasting increased serum total bilirubin with a mean of $0.20 \pm 0.06 \mathrm{mg} / \mathrm{dl}(p=0.0004)$ and decreased insulin requirements with a mean of $1.16 \pm 0.14 \mathrm{IU} / \mathrm{h}(p<$ 0.0001 ) as compared with feeding. Over the total intervention day, the cumulative insulin dose was similar between both groups ( $p=0.15$, Additional table 3$)$.

Plasma $\mathrm{BOH}$ and serum IGF-I concentrations were significantly affected by the intervention (both interaction $p<0.0001)$. A 12-h fasting period increased $\mathrm{BOH}$ with a mean of $0.47 \pm 0.07 \mathrm{mmol} / \mathrm{l}(p<0.0001)$ and decreased IGF-I with a mean of $13.9 \pm 1.6 \mathrm{ng} / \mathrm{ml}(p<0.0001)$ as compared with the 12-h feeding period. Serum urea was unaffected by the intervention (interaction $p=0.68$ ).

Consecutive point-of-care $\mathrm{BOH}$ measurements confirmed a significantly different time profile for both randomization groups (interaction $p<0.0001$ ). $\mathrm{BOH}$ was already increased from $4 \mathrm{~h}$ fasting onwards and was suppressed from $4 \mathrm{~h}$ feeding onwards (mean delta $\mathrm{BOH}$ after $4 \mathrm{~h}$ of fasting $0.1 \pm 0.37 \mathrm{mmol} / \mathrm{l}$, after $4 \mathrm{~h}$ of feeding $-0.1 \pm 0.23 \mathrm{mmol} / \mathrm{l}, p=0.0001$, Fig. 4). BOH remained elevated throughout the 12-h fasting interval (mean $\mathrm{BOH}$ after $4 \mathrm{~h}$ of fasting $0.4 \pm 0.04 \mathrm{mmol} / \mathrm{L}$, after $8 \mathrm{~h}$ of fasting $0.4 \pm 0.04 \mathrm{mmol} / \mathrm{l}$, after $12 \mathrm{~h}$ of fasting $0.5 \pm 0.05$ $\mathrm{mmol} / \mathrm{l})$. As compared to $4 \mathrm{~h}$ fasting, $\mathrm{BOH}$ did not significantly change over the remainder of the 12-h fasting interval (mean delta between $4 \mathrm{~h}$ and $8 \mathrm{~h}$ of fasting $0.0 \pm$ $0.04 \mathrm{mmol} / \mathrm{l}$, mean delta between $4 \mathrm{~h}$ and $12 \mathrm{~h}$ of fasting $0.0 \pm 0.05 \mathrm{mmol} / \mathrm{l}, p 0.76$ ). 


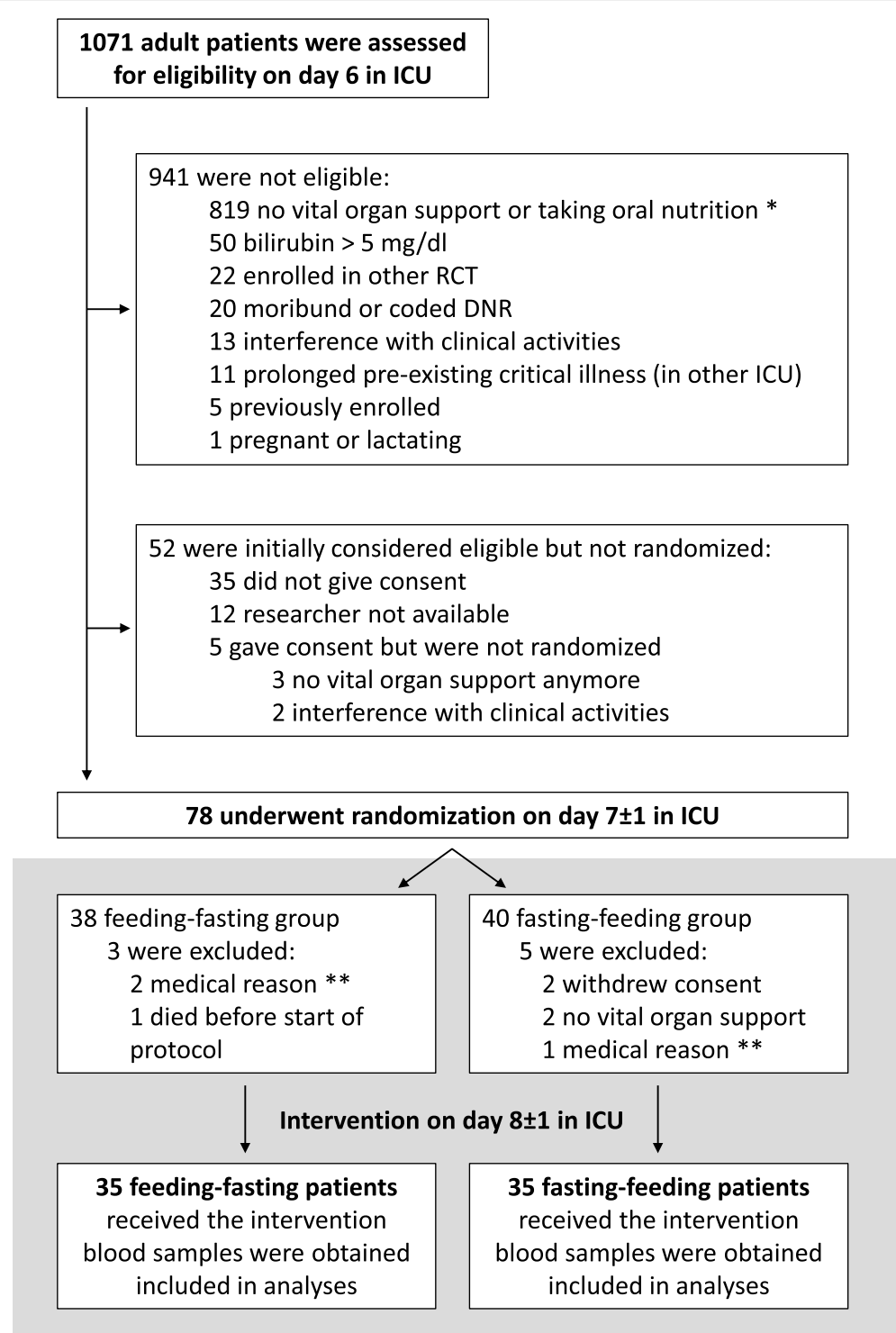

Fig. 1 Enrollment and randomization. Reasons for ineligibility and non-inclusion of eligible patients are listed. Ultimately, 35 patients per randomization group completed the study protocol, hence 70 patients in total. *Vital organ support was defined as dependency on mechanical ventilation and mechanical and/or pharmacological hemodynamic support. **Medical reasons include high glucose need to treat hypernatremia $(n=2)$ or multiple hypoglycemic episodes between randomization and start of the protocol $(n=1)$. ICU, intensive care unit; RCT, randomized controlled trial; DNR, do not resuscitate

Autophagy activation in whole blood and white blood cells mRNA expression of autophagy-related genes was not significantly affected by the intervention, neither in whole blood (interaction $p 0.08$ for $p 62,0.75$ for atg3, 0.51 for $\operatorname{atg} 5$, and 0.64 for $\operatorname{atg} 7$ ), nor in isolated white blood cells (interaction $p 0.62$ for $p 62,0.17$ for atg3, 0.29 for $\operatorname{atg} 5$, and 0.13 for $\operatorname{atg} 7$ ) (Fig. 5). Also in healthy volunteers, there was no effect of nutrition on gene expression in whole blood when comparing the fasted state to the fed state ( $p$ values for within-subject effect of time 0.40 for $p 62 ; 0.58$ for atg3; 0.07 for atg5; 0.58 for atg7). In the isolated white blood cells of controls, fasting slightly induced expression of atg3 with an increase of $6.7 \%$, but no effect was observed on the other autophagy genes ( $p$ values for within subjects effect of time 0.83 for p62; 0.04 for atg3; 0.94 for atg5; 0.30 for atg7).

Protein expression of key autophagy markers was not significantly affected by the intervention (interaction $p$ 0.38 for p62, 0.56 for the ratio of LC3 II/LC3 I, 0.12 for LC3 I, and 0.37 for LC3 II) (Fig. 5). Also in healthy volunteers, protein levels of these markers were not different between the fasted and the fed state ( $p$ values for within subjects effect of time 0.91 for p62, 0.46 for the ratio of LC3 II/LC3 I, 0.52 for LC3 I, and 0.63 for LC3 II). 


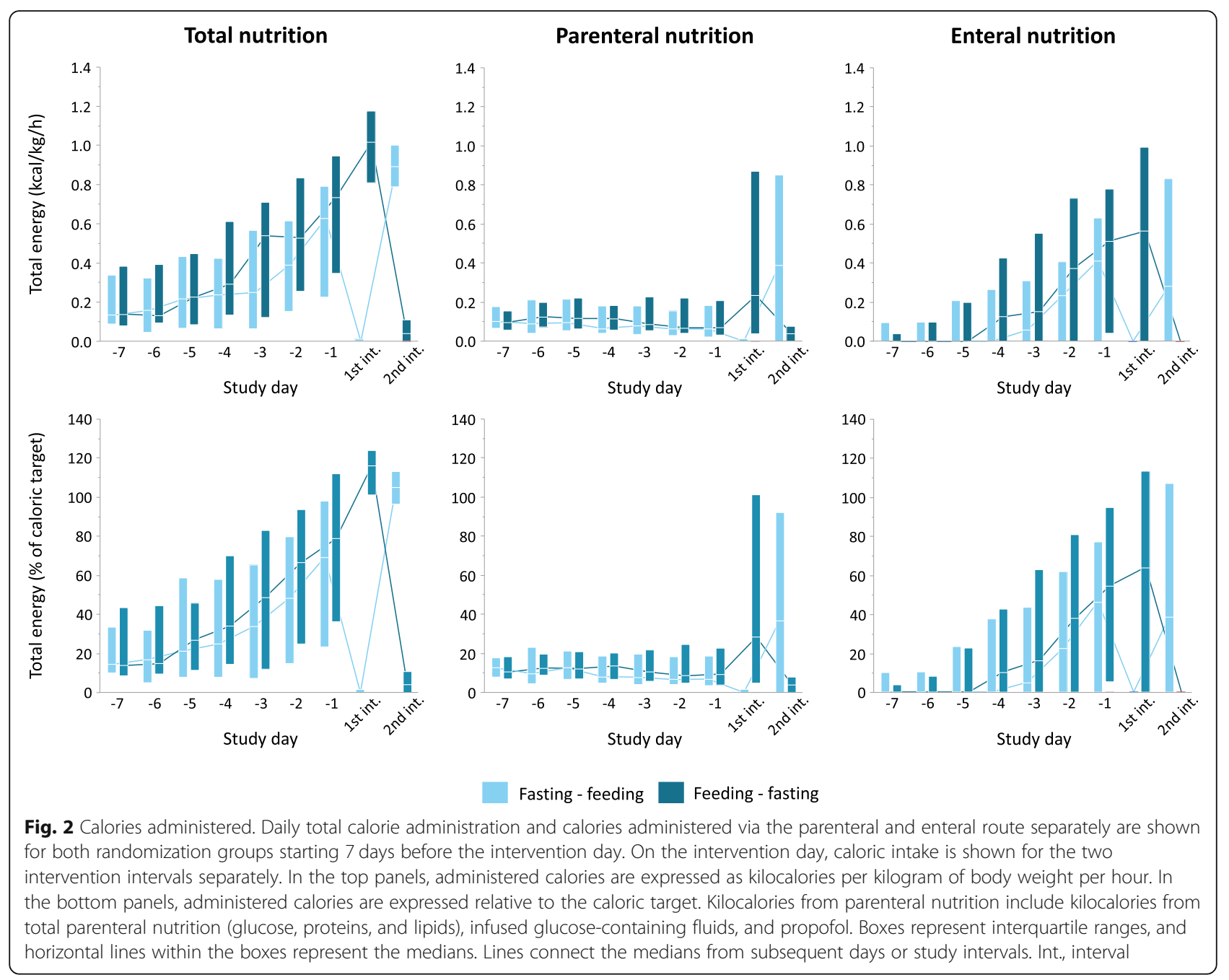

\section{Morbidity and mortality}

During the 12-h fasting interval, severe hypoglycemia occurred in 3 patients. All events occurred under treatment with intravenous insulin; hence, no patient suffered from spontaneous hypoglycemia. In two of the events, unlike prescribed in the study protocol, the insulin dose was not reduced at the start of the fasting interval. All hypoglycemic events were countered by parenteral glucose administration. None of the patients died within $24 \mathrm{~h}$ after the hypoglycemic event. In the first week after the intervention, the incidence of new infections and persistent need for hemodynamic support, mechanical respiratory support, and renal replacement therapy were comparable between both randomization groups (Additional table 3). Mortality within the first week after the intervention and ICU mortality were comparable between both randomization groups. Ninetyday mortality was higher in the feeding-fasting group than in the fasting-feeding group ( $p$ 0.003). Causes of death are reported in Additional table 4.

\section{Discussion}

In this randomized crossover study of 70 prolonged critically ill patients, we found that 12 -h nutrient restriction initiated a metabolic fasting response, as evidenced by a rise in serum total bilirubin and a decrease in insulin needs to maintain normal blood glucose concentrations. Also, a rise in plasma $\mathrm{BOH}$ and a decrease in serum IGF-I was observed with a 12-h fast, whereas serum urea was unaffected. The increase in blood $\mathrm{BOH}$ was already present after $4 \mathrm{~h}$ of fasting, and prolongation of the fasting period did not result in a further increase. Neither in healthy controls nor in prolonged critically ill patients did a 12-h fasting affect autophagy-related gene and protein expression in whole blood or isolated white blood cells.

Recent RCTs have shown that accepting an early macronutrient deficit by withholding $\mathrm{PN}$ in the acute phase of critical illness may be beneficial, which suggests that activating a fasting response may be protective in critically ill patients $[4,5]$. Beyond the acute phase, however, 


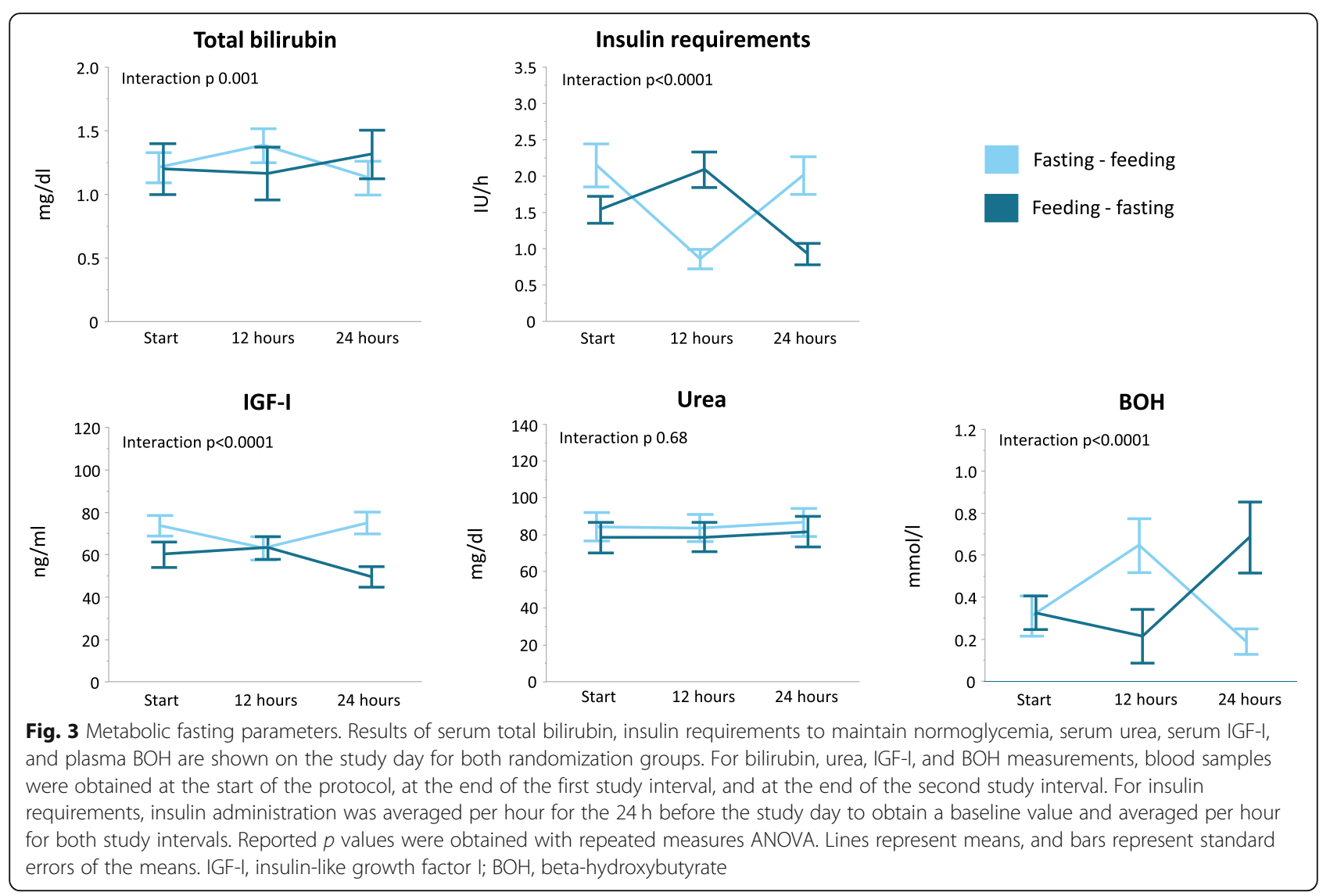

BOH

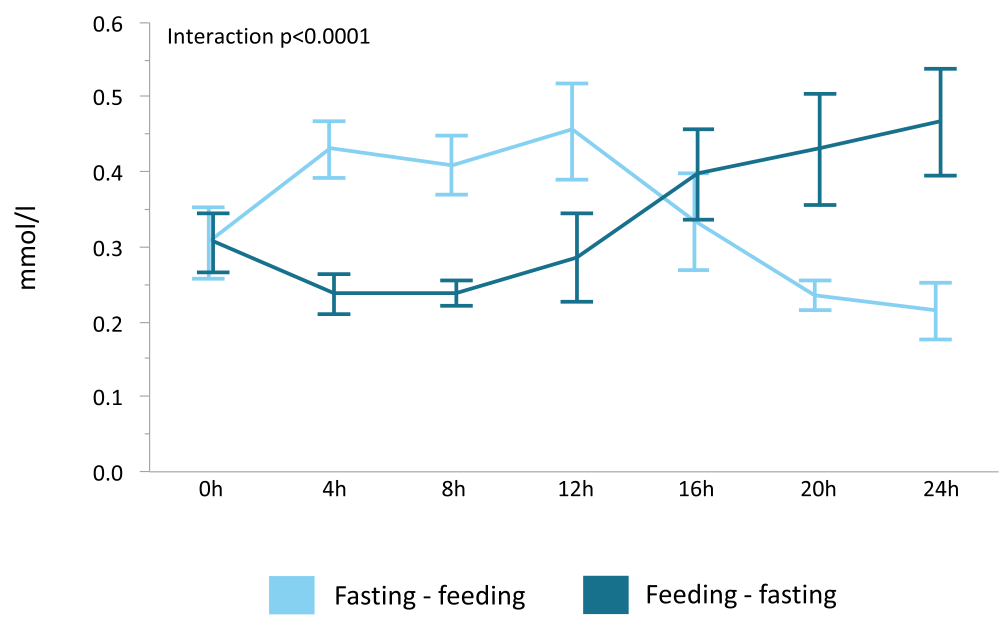

Fig. $4 \mathrm{POC}$ ketone meters. Results of $\mathrm{BOH}$ measurements with use of a point of care ketone meter during the study day. $\mathrm{BOH}$ was measured every $4 \mathrm{~h}$. Results are shown for both randomization groups. $0 \mathrm{~h}$ marks the start of the study day, $12 \mathrm{~h}$ marks the end of the first study interval, and $24 \mathrm{~h}$ marks the end of the second study interval. The reported $p$ value was obtained using repeated measures ANOVA. Lines represent means, and bars represent standard errors of the means. BOH, beta-hydroxybutyrate 


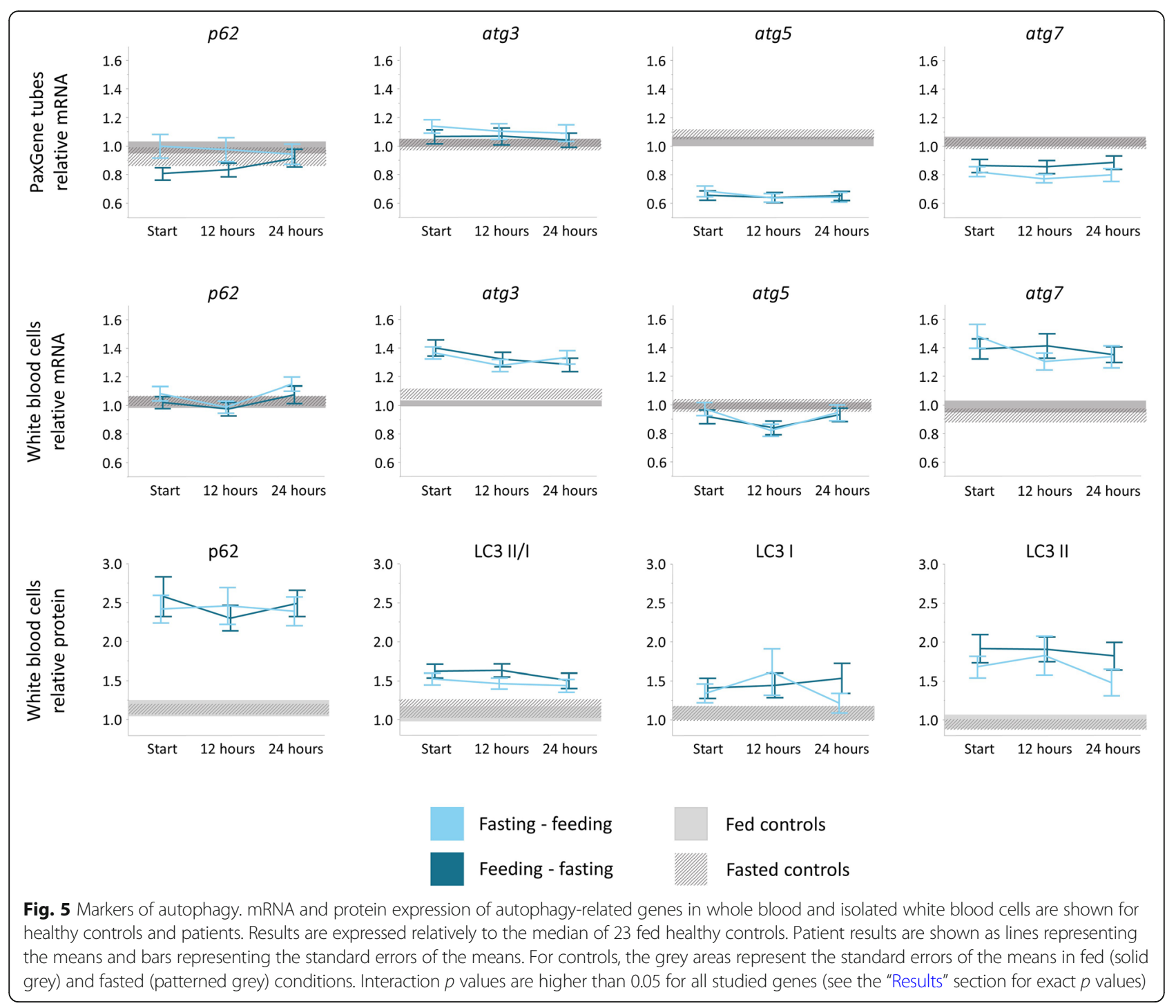

prolonged starvation may support ongoing catabolism, which is unwanted. Recent studies in other models have suggested that fasting-induced benefits can also be brought about by so-called fasting-mimicking diets, which alternate periods of restricted feeding with periods of unlimited feeding [19]. Indeed, in healthy adults and mice, fasting-mimicking diets improved the metabolic profile, inflammation, and regenerative markers, which in animal models also prevented age-related diseases and increased lifespan [19]. Interestingly, in an animal model, also in the fed phase of the fasting-mimicking diet, ketone concentrations were higher than with the ad libitum diet, which suggests that fasting responses could be partially maintained in the fed state [19]. The current study opens perspectives for the further design and clinical validation of a fasting-mimicking diet for critically ill patients. Indeed, our pilot study demonstrated that 12-h feeding interruption initiated a metabolic fasting response in prolonged critically ill patients, with increases in serum bilirubin and blood/plasma $\mathrm{BOH}$ and decreases in insulin need and IGF-I. Although the observed differences were relatively small, fasting-mimicking diets in other settings have shown similarly small but sustained changes while providing clinical benefit $[19,29]$. In our study, serum urea was not affected, which may be explained by the relatively brief fasting period. Indeed, in healthy adults, urea concentrations only rose after a considerably longer fasting episode [18]. On the other hand, urea may not be a good marker of a metabolic fasting response in critically ill patients, in whom artificial feeding may also result in increased ureagenesis through catabolism of the supplementary provided amino acids [30]. Future studies should investigate whether time-restricted feeding by daily interruption of artificial feeding for $12 \mathrm{~h}$ can maintain a sustained metabolic fasting response and whether this allows to induce autophagy in tissues of interest and improves clinical 
outcomes. Although speculative, another potential advantage of time-restricted feeding could be a better preservation of circadian rhythm when the fasting interval would be held during the night $[31,32]$. Time-restricted feeding also has potential downsides, however, including an increased risk of prolonged underfeeding if nutrition cannot be proportionally increased in the feeding interval or else a potentially increased risk of feeding intolerance, and potentially larger fluctuations in blood glucose, which have been associated with worse outcome [33, 34].

In contrast to previous studies suggesting impaired ketogenesis in critical illness, our results indicate increased ketogenesis already after $4 \mathrm{~h}$ of fasting [22, 23]. Although speculative, prevention of hyperglycemia in our study may have played a role. Indeed, high circulating glucose concentrations can suppress lipolysis, and this condition was prevented by continuous intravenous insulin administration [35]. On the other hand, insulin also suppresses lipolysis and ketogenesis [36]. However, our group previously demonstrated in a subanalysis of a RCT that tight glucose control improved insulin sensitivity in skeletal muscle of prolonged critically ill patients, but not in liver [37]. Hence, in prolonged critically ill patients, tight glucose control was realized with similar serum insulin concentrations as in patients in whom moderate hyperglycemia was tolerated, in the presence of severe hepatic insulin resistance.

In both healthy volunteers and prolonged critically ill patients, we did not find any consistent impact of a 12-h fasting episode on gene and protein expression of autophagyrelated genes in whole blood and isolated white blood cells. This contrasts with a previous study that showed activation of autophagy in skeletal muscle by withholding PN until 1 week after ICU admission, which correlated with improved muscle function [14]. This apparent discrepancy may point to tissue-dependent changes in autophagy activation. Indeed, in mice, starvation-induced autophagy activation differed among organs, with a more distinct early induction in muscle as compared to other tissues [38]. Furthermore, the metabolism of white blood cells has been shown to be relatively resistant to starvation in healthy adults [39]. Hence, our data may suggest that blood samples are not a good surrogate for autophagy at the level of other tissues, although this requires further investigation in a study that compares different tissues. Alternatively, the fasting interval may have been too short to detect fastinginduced autophagy. However, we also did not find any impact of fasting on autophagic markers in healthy volunteers. Moreover, autophagy has been shown to be activated by 12-h fasting in muscle of healthy animals, although one should be cautious when extrapolating results from animals to humans [40].

Unexpectedly, the study revealed a significantly different 90-day mortality between both randomization groups. Although we cannot exclude an impact of randomization, it appears unlikely that a feeding study only lasting $24 \mathrm{~h}$ would affect mortality on a longer term, as none of the large RCTs on nutritional management in the ICU has observed any impact of nutrition on survival [33]. Moreover, short-term morbidity and mortality at day 7 and in ICU were similar in both groups. Finally, since all patients received the same interventions in a crossover design at day 8 in ICU, it seems unlikely that the order of such intervention would have affected clinical outcome only on the long term. Instead, it could be that patients in the feeding-fasting group may have been sicker than the fasting-feeding group, although this was not captured by the SOFA scores.

The study has important strengths. We demonstrated a clear initiation of a metabolic fasting response during a 12-h fasting period, which was consistently observed on different metabolic parameters in a crossover design. Our study inherently has some limitations. The sample size was relatively small, and the study may have been underpowered for some secondary endpoints. Second, we only investigated a 12-h fasting interval, whereby we do not have data on longer fasting intervals that may induce a more pronounced metabolic fasting response. However, the duration of fasting was a pragmatic choice between a sufficiently long period of fasting while leaving an acceptable time to feed. Third, differences in concomitant medication between the two study periods may theoretically have impacted certain outcome parameters. However, this risk is minimal in a crossover study, since we expect that significant medication adaptations on the intervention day with immediate impact on the studied parameters would be rare. Fourth, we calculated the daily caloric target using a formula. Although common practice, this value does not necessarily match the energy expenditure measured by indirect calorimetry. Finally, to reduce the metabolic burden of administering the daily calculated nutrient dose over only $12 \mathrm{~h}$, we only administered half of the calculated daily nutrition dose in the 12-h feeding interval.

\section{Conclusion}

A 12-h macronutrient interruption was able to initiate a metabolic fasting response in prolonged critically ill patients, with increases in bilirubin and ketones and decreases in insulin needs and IGF-I. Twelve hours fasting did not affect markers of autophagy in isolated white blood cells and whole blood samples of prolonged critically ill patients or healthy volunteers, and thus, blood samples may not be a good readout to assess autophagy at the level of other tissues. The current study opens perspectives for the development of a fasting-mimicking diet for critically ill patients, which could consist of repeated blocks of $12 \mathrm{~h}$ of feeding alternated with $12 \mathrm{~h}$ of fasting. Future research should investigate whether such diet is able to maintain a sustained fasting response and whether this delivers clinical benefit. 


\section{Supplementary information}

Supplementary information accompanies this paper at https://doi.org/10. 1186/s13054-020-02987-3.

Additional file 1. (VanDyck-ICU-FM-additional_table1). Calculation of caloric target. Description of data: Formula used to calculate the caloric target.

Additional file 2. (VanDyck-ICU-FM-additional_table2). Formulas of enteral and parenteral nutrition. Description of data: Formulas of enteral and parenteral nutrition used in the study.

Additional file 3. (VanDyck-ICU-FM-additional_table3). Nutrition on the intervention day and clinical endpoints. Description of data: Nutrition on the intervention day combined for both intervention windows. ICUrelated complications for 7 days after the study day, or until ICU discharge if the ICU stay was shorter, and for short- and long-term mortality.

Additional file 4. (VanDyck-ICU-FM-additional_table4). Cause of death for 90 day mortality. Description of data: Cause of death for patients who died within 90 days after randomization

\section{Abbreviations}

APACHE II: Acute Physiology and Chronic Health Evaluation II; BMI: Body mass index; $\mathrm{BOH}$ : Beta-hydroxybutyrate; EN: Enteral nutrition; ICU: Intensive care unit; IGF-I: Insulin-like growth factor I; NRS: Nutritional Risk Screening; PN: Parenteral nutrition; POC: Point of care; RCT: Randomized controlled trial; SOFA: Sequential Organ Failure Assessment

\section{Acknowledgements}

We would like to thank Nathalie Van Aerde for her practical help.

\section{Authors' contributions}

GVDB, JG, and MPC designed the study. LVD, JG, and MPC collected, analyzed, and interpreted the data and drafted the first manuscript. IV, AW, AS, and ID assisted with data collection. LM and PJW were in charge of the data management. All authors read and approved the final manuscript.

\section{Funding}

This work was supported by the Research Foundation-Flanders (FWO), Belgium (predoctoral research fellowship to L. Van Dyck, fundamental clinical research fellowship to M. Casaer); by the Methusalem program of the Flemish government (through the University of Leuven to G. Van den Berghe and I. Vanhorebeek, METH14/06); by European Research Council Advanced Grants (AdvG-2012-321670 and AdvG-2017-785809) to G. Van den Berghe; by the University Hospitals Leuven (postdoctoral research fellowship grant to J. Gunst); and by the KU Leuven (C2 project funding to M. Casaer and J. Gunst, Start-Up grant to M. Casaer)

\section{Availability of data and materials}

Data sharing will be considered only on a collaborative basis with the principal investigators, after evaluation of the proposed study protocol and statistical analysis plan.

\section{Ethics approval and consent to participate}

The Ethical Committee Research UZ/KU Leuven approved the protocol and consent forms (S59328). All patients or their legal representatives provided written informed consent before randomization.

\section{Consent for publication}

Not applicable.

\section{Competing interests}

None of the authors has any conflict of interest to report. Nova Biomedical and Menarini Diagnostics kindly provided the point-of-care ketone meters and accompanying analytical disposables, but were not involved in design of the study, in data collection, data analysis, writing of the manuscript, or in the decision to submit for publication.

\section{Author details}

'Department of Cellular and Molecular Medicine, Clinical Division and Laboratory of Intensive Care Medicine, KU Leuven, Herestraat 49, B-3000
Leuven, Belgium. ${ }^{2}$ Medical Intensive Care Unit, Department of Internal Medicine, University Hospitals Leuven, Leuven, Belgium.

Received: 18 February 2020 Accepted: 12 May 2020

Published online: 24 May 2020

\section{References}

1. Alberda C, Gramlich L, Jones N, Jeejeebhoy K, Day AG, Dhaliwal R, et al. The relationship between nutritional intake and clinical outcomes in critically ill patients: results of an international multicenter observational study. Intensive Care Med. 2009:35(10):1728-37.

2. Weijs PJ, Stapel SN, de Groot SD, Driessen RH, de Jong E, Girbes AR, et al. Optimal protein and energy nutrition decreases mortality in mechanically ventilated, critically ill patients: a prospective observational cohort study. JPEN J Parenter Enteral Nutr. 2012;36(1):60-8.

3. Artinian $V$, Krayem H, DiGiovine B. Effects of early enteral feeding on the outcome of critically ill mechanically ventilated medical patients. Chest. 2006:129(4):960-7

4. Casaer MP, Mesotten D, Hermans G, Wouters PJ, Schetz M, Meyfroidt G, et al. Early versus late parenteral nutrition in critically ill adults. N Engl J Med. 2011;365(6):506-17

5. Fivez T, Kerklaan D, Mesotten D, Verbruggen S, Wouters PJ, Vanhorebeek et al. Early versus late parenteral nutrition in critically ill children. N Engl J Med. 2016:374(12):1111-22.

6. Rice TW, Wheeler AP, Thompson BT, Steingrub J, Hite RD, Moss M, et al. Initial trophic vs full enteral feeding in patients with acute lung injury: the EDEN randomized trial. JAMA. 2012;307(8):795-803.

7. Arabi YM, Aldawood AS, Haddad SH, Al-Dorzi HM, Tamim HM, Jones G, et al. Permissive underfeeding or standard enteral feeding in critically ill adults. N Engl J Med. 2015;372(25):2398-408.

8. Target Investigators ftACTG, Chapman M, Peake SL, Bellomo R, Davies A Deane $A$, et al. Energy-dense versus routine enteral nutrition in the critically ill. N Engl J Med. 2018;379(19):1823-34.

9. Allingstrup MJ, Kondrup J, Wiis J, Claudius C, Pedersen UG, Hein-Rasmussen $R$, et al. Early goal-directed nutrition versus standard of care in adult intensive care patients: the single-centre, randomised, outcome assessorblinded EAT-ICU trial. Intensive Care Med. 2017:43(11):1637-47.

10. Casaer MP, Wilmer A, Hermans G, Wouters PJ, Mesotten D, Van den Berghe $G$. Role of disease and macronutrient dose in the randomized controlled EPaNIC trial: a post hoc analysis. Am J Respir Crit Care Med. 2013;187(3): 247-55.

11. Vanhorebeek I, Verbruggen S, Casaer MP, Gunst J, Wouters PJ, Hanot J, et al. Effect of early supplemental parenteral nutrition in the paediatric ICU: a preplanned observational study of post-randomisation treatments in the PEPaNIC trial. Lancet Respir Med. 2017;5(6):475-83.

12. Harvey SE, Parrott F, Harrison DA, Bear DE, Segaran E, Beale R, et al. Trial of the route of early nutritional support in critically ill adults. N Engl J Med. 2014:371(18):1673-84

13. Reignier J, Boisramé-Helms J, Brisard L, Lascarrou JB, Ait Hssain A, Anguel N, et al. Enteral versus parenteral early nutrition in ventilated adults with shock: a randomised, controlled, multicentre, open-label, parallel-group study (NUTRIREA-2). Lancet. 2018:391(10116):133-43.

14. Hermans G, Casaer MP, Clerckx B, Güiza F, Vanhullebusch T, Derde S, et al. Effect of tolerating macronutrient deficit on the development of intensivecare unit acquired weakness: a subanalysis of the EPaNIC trial. Lancet Respir Med. 2013;1(8):621-9.

15. Goossens C, Weckx R, Derde S, Dufour T, Vander Perre S, Pauwels L, et al. Adipose tissue protects against sepsis-induced muscle weakness in mice: from lipolysis to ketones. Crit Care. 2019;23(1):236

16. Van Dyck L, Casaer MP, Gunst J. Autophagy and its implications against early full nutrition support in critical illness. Nutr Clin Pract. 2018:33(3):339-47.

17. Gunst J. Recovery from critical illness-induced organ failure: the role of autophagy. Crit Care. 2017:21(1):209.

18. Cahill GF Jr. Starvation in man. Clin Endocrinol Metab. 1976;5(2):397-415

19. Brandhorst S, Choi IY, Wei M, Cheng CW, Sedrakyan S, Navarrete G, et al. A periodic diet that mimics fasting promotes multi-system regeneration, enhanced cognitive performance, and healthspan. Cell Metab. 2015;22(1): 86-99.

20. Curtis E, Litwic A, Cooper C, Dennison E. Determinants of muscle and bone aging. J Cell Physiol. 2015;230(11):2618-25. 
21. Preiser JC, Ichai C, Orban JC, Groeneveld AB. Metabolic response to the stress of critical illness. Br J Anaesth. 2014;113(6):945-54.

22. Ohtoshi M, Jikko A, Asano M, Uchida K, Ozawa K, Tobe T. Ketogenesis during sepsis in relation to hepatic energy metabolism. Res Exp Med (Berl). 1984;184(4):209-19.

23. Watters JM, Wilmore DW. Role of catabolic hormones in the hypoketonaemia of injury. Br J Surg. 1986;73(2):108-10.

24. Cahill GF Jr, Herrera MG, Morgan AP, Soeldner JS, Steinke J, Levy PL, et al. Hormone-fuel interrelationships during fasting. J Clin Invest. 1966;45(11): 1751-69.

25. Whitmer DI, Gollan JL. Mechanisms and significance of fasting and dietary hyperbilirubinemia. Semin Liver Dis. 1983;3(1):42-51.

26. Thissen JP, Ketelslegers JM, Underwood LE. Nutritional regulation of the insulin-like growth factors. Endocr Rev. 1994;15(1):80-101.

27. Singer M, Deutschman CS, Seymour CW, Shankar-Hari M, Annane D, Bauer $M$, et al. The Third International Consensus Definitions for Sepsis and Septic Shock (Sepsis-3). JAMA. 2016;315(8):801-10.

28. Erdfelder E, Faul F, Buchner A. GPOWER: a general power analysis program. Behav Res Methods Instrum Comput. 1996;28:1-11.

29. Wilkinson MJ, Manoogian ENC, Zadourian A, Lo H, Fakhouri S, Shoghi A, et al. Ten-hour time-restricted eating reduces weight, blood pressure, and atherogenic lipids in patients with metabolic syndrome. Cell Metab. 2020; 31(1):92-104 e5.

30. Gunst J, Vanhorebeek I, Casaer MP, Hermans G, Wouters PJ, Dubois J, et al, Impact of early parenteral nutrition on metabolism and kidney injury. J Am Soc Nephrol. 2013;24(6):995-1005.

31. Oike H, Oishi K, Kobori M. Nutrients, clock genes, and chrononutrition. Curr Nutr Rep. 2014;3:204-12.

32. Korompeli A, Muurlink $\mathrm{O}$, Kavrochorianou N, Katsoulas T, Fildissis $\mathrm{G}$, Baltopoulos G. Circadian disruption of ICU patients: a review of pathways, expression, and interventions. J Crit Care. 2017;38:269-77.

33. Singer $P$, Blaser AR, Berger MM, Alhazzani W, Calder PC, Casaer MP, et al. ESPEN guideline on clinical nutrition in the intensive care unit. Clin Nutr. 2019;38(1):48-79.

34. Evans DC, Forbes R, Jones C, Cotterman R, Nioku C, Thongrong C, et al. Continuous versus bolus tube feeds: does the modality affect glycemic variability, tube feeding volume, caloric intake, or insulin utilization? Int J Crit IIIn Inj Sci. 2016;6(1):9-15.

35. Langouche L, Vanhorebeek I, Van den Berghe G. Therapy insight: the effect of tight glycemic control in acute illness. Nat Clin Pract Endocrinol Metab. 2007;3(3):270-8.

36. Duncan RE, Ahmadian M, Jaworski K, Sarkadi-Nagy E, Sul HS. Regulation of lipolysis in adipocytes. Annu Rev Nutr. 2007;27:79-101.

37. Langouche L, Vander Perre S, Wouters PJ, D'Hoore A, Hansen TK, Van den Berghe $\mathrm{G}$. Effect of intensive insulin therapy on insulin sensitivity in the critically ill. J Clin Endocrinol Metab. 2007:92(10):3890-7.

38. Mizushima N, Yamamoto A, Matsui M, Yoshimori T, Ohsumi Y. In vivo analysis of autophagy in response to nutrient starvation using transgenic mice expressing a fluorescent autophagosome marker. Mol Biol Cell. 2004; 15(3):1101-11.

39. Pietrocola F, Demont Y, Castoldi F, Enot D, Durand S, Semeraro M, et al. Metabolic effects of fasting on human and mouse blood in vivo. Autophagy. 2017;13(3):567-78.

40. Grumati P, Coletto L, Sabatelli P, Cescon M, Angelin A, Bertaggia E, et al. Autophagy is defective in collagen $\mathrm{VI}$ muscular dystrophies, and its reactivation rescues myofiber degeneration. Nat Med. 2010;16(11):1313-20.

\section{Publisher's Note}

Springer Nature remains neutral with regard to jurisdictional claims in published maps and institutional affiliations.

Ready to submit your research? Choose BMC and benefit from:

- fast, convenient online submission

- thorough peer review by experienced researchers in your field

- rapid publication on acceptance

- support for research data, including large and complex data types

- gold Open Access which fosters wider collaboration and increased citations

- maximum visibility for your research: over $100 \mathrm{M}$ website views per year

At BMC, research is always in progress.

Learn more biomedcentral.com/submissions 